\title{
Les chemins de Pierre Bergounioux, S. Coyault, M. T. Jacquet (dir.)
}

\section{Simonetta Valenti}

\section{Q OpenEdition \\ 1 Journals}

\section{Edizione digitale}

URL: http://journals.openedition.org/studifrancesi/10066

DOI: 10.4000/studifrancesi. 10066

ISSN: 2421-5856

\section{Editore}

Rosenberg \& Sellier

\section{Edizione cartacea}

Data di pubblicazione: 1 août 2017

Paginazione: 402-403

ISSN: 0039-2944

\section{Notizia bibliografica digitale}

Simonetta Valenti, «Les chemins de Pierre Bergounioux, S. Coyault, M. T. Jacquet (dir.)», Studi Francesi

[Online], 182 (LXI | II) | 2017, online dal 01 août 2017, consultato il 07 janvier 2021. URL: http:// journals.openedition.org/studifrancesi/10066 ; DOI: https://doi.org/10.4000/studifrancesi. 10066

Questo documento è stato generato automaticamente il 7 janvier 2021.

\section{(c) (i) $\odot$}

Studi Francesi è distribuita con Licenza Creative Commons Attribuzione - Non commerciale - Non opere derivate 4.0 Internazionale. 


\title{
Les chemins de Pierre Bergounioux, S. Coyault, M. T. Jacquet (dir.)
}

\author{
Simonetta Valenti
}

\section{NOTIZIA}

Les chemins de Pierre Bergounioux, sous la direction de Sylviane CoyauLT, Marie Thérèse JACQUET, Macerata, Quodlibet Studio, 2016, 351 pp.

1 Dedicato alla figura e all'opera di Pierre Bergounioux, il presente volume è preceduto da un Avant-propos (pp. 9-17), nel quale Sylviane coyault offre al lettore una chiara sintesi del percorso creativo dello scrittore.

2 Nel suo saggio, Marie Thérèse JACQUET presenta l'opera di Bergounioux come pervasa dalla descrizione lenta, metodica, ripetitiva di una quotidianità che, dietro l'apparente banalità, cela istanti di autentica rivelazione, descritti in uno stile elegante, colto $\mathrm{e}$ meditato.

Dal canto suo, Mathilde BARRABAND mostra come, nell'opera dello scrittore, sapere e letteratura, spiegazione e narrazione si siano sempre intrecciati, dando vita a un tipo di racconto che va dal romanzo all'autobiografia, dall'allegoria all'enquête individuale.

4 Studiando in parallelo L'orphelin, La casse e La ligne, Manet van MONTFRANS si sofferma sulle diverse forme che il récit de filiation tende ad assumere nell'opera di Bergounioux, all'interno della quale esso costituisce una delle modalità che si offrono al narratore per giungere a una maggiore conoscenza di sé.

5 Giusi Alessandra FALCO indaga il tema dell'assenza, onnipresente nella produzione di Bergounioux, ma certamente centrale ne L'orphelin, dove essa tende a occupare uno spazio sempre maggiore, fino a diventare parte integrante della narrazione stessa.

Alain SCHAFFNER si concentra invece sul significato del récit d'enfance ne La maison rose, sottolineando come esso articoli la riscoperta del passato familiare, mediante una narrazione al tempo stesso lineare, discontinua e circolare. 
7 Nel suo contributo, Aurélie ADLER illustra il tema della caccia in Catherine e Chasseur à la manque quale metafora della ricerca della conoscenza e della scrittura che, nelle opere di Bergounioux, assume i tratti del mito originario.

Nel suo contributo, Anna Isabella SQUARZINA si sofferma ad analizzare Trente mots, un testo di difficile definizione che presenta, ricorrendo alla struttura del glossario, il tentativo paradossale, da parte di Bergounioux, di approfondire la difficoltà intrinseca del linguaggio a rendere la vita.

Grazia TAMBURINI cerca di ricostruire la poetica di Bergounioux, prendendo in esame il saggio Le style comme expérience. Incapace di imprimere un senso alla propria esistenza, l'autore limosino ricorre alle parole e ai saperi che esse veicolano, allo scopo di comprendere il mondo che lo circonda, ma anche i drammi avvenuti nel passato, le cui ferite domandano di essere lenite nel presente attraverso la scrittura.

Come per Bourdieu, anche per Bergounioux, la definizione dello stile oscilla costantemente tra l'appartenenza a una collettività e la ricerca di una individualità. Questa è la ragione per la quale - secondo Laurent DEMANZE - lo scrittore insiste nelle sue opere sulla doppia valenza della scrittura: storica e individuale.

11 Dominique VIART sottolinea la portata fondante del passato nell'opera di Pierre Bergounioux che, soprattutto nei racconti e nei testi brevi, ricorre a lunghe introduzioni storiche nelle quali ripercorre l'origine arcaica dei fenomeni, per giungere al punto focale che suscita il suo interesse nel presente. La visione storica si arricchisce allora di elementi geografici e sociologici, che concorrono a forgiare «la geste de l'Histoire en tant que telle» (p. 140).

Interessandosi al dittico formato da B-17G, da un lato, e da Le récit absent e Le baiser de la sorcière, dall'altro, Jean-Bernard VRAY studia il rapporto che questi testi instaurano con l'immagine fotografica. Essa è funzionale, da una parte, ad ancorare l'universo diegetico alla Storia e, dall'altro, a mostrarne la portata soggettiva, attraverso lo sguardo retrospettivo dello scrittore, che coglie i capitoli oscuri del passato, mettendoli a nudo mediante la scrittura.

In La mort de Brune, Bergounioux ci offre - a detta di Pierre D'ALMEIDA - la sua personale interpretazione della storia. Con l'assassinio del maresciallo limosino Brune (1815), rappresentato in una tela che giganteggia nel museo di Brive, i grandi ideali della Rivoluzione francese sembrano essersi infranti e la Storia irrigidita in una ferocia inaudita, di cui gli abitanti sono destinati ad avvertire per sempre l'angoscia. Per tale ragione, in Bergounioux la scrittura diviene lo strumento per sottrarre la verità dell'istante, tanto all'oblio del passato, quanto all'accelerazione scomposta del presente.

Rileggendo La fin du monde en avançant, Valerio CORDINER analizza ciascuna delle quattro parti. In esse, Bergounioux dà vita a una riflessione filosofica, mostrando come la Rivoluzione francese si sia realizzata concretamente attraverso violenza e distruzione, che hanno spazzato via ogni speranza di futuro per gli abitanti delle campagne, i quali, a partire dagli anni Sessanta del Novecento, hanno perso definitivamente la loro identità. L'alienazione della società contemporanea, causata dalla smaterializzazione del lavoro, ha così creato masse anonime di lavoratori, condannati a vivere nelle periferie delle metropoli, secondo i ritmi forsennati della produzione. Di qui l'esigenza di riaffermare la fondatezza dell'attività artistica - e più specificamente della 
letteratura - quale forma radicale di resistenza al declino che minaccia il presente, ancorandolo alla memoria della Repubblica e dei suoi valori.

Nel suo contributo, Jochen MECKE mostra come, a partire da Miette, Bergounioux sia passato a forme di narrazione che privilegiano figure apparentemente insignificanti, la cui vicenda è raccontata attraverso una successione discontinua di immagini giustapposte, funzionali a rendere sensibile la dimensione ciclica del tempo.

Nell'attuale società globale, connotata dalla standardizzazione del gusto, l'uomo sembra vedersi negata la possibilità di fare una vera esperienza del reale e ciò sarebbe all'origine, per Bergounioux, della crisi della stessa letteratura. Paolo TAMASSIA osserva allora come, per ridare significato al fatto letterario, occorra, secondo l'autore francese, ridare consistenza alla quotidianità, intesa quale momento imprescindibile dell'esperienza esistenziale dell'uomo contemporaneo.

Studiando le tesi illustrate in Une chambre en Hollande, Agir, écrire e Le récit absent, Stéphane CHAUDIER si sofferma sulle numerose contraddizioni che paiono insinuarsi nel pensiero di Bergounioux. La ragione profonda di esse - conclude lo studioso - si trova nondimeno nel divenire storico stesso, la cui dialettica mostra il superamento costante dell'ordine della ragione da parte della realtà e la conseguente esigenza di riavviare continuamente l'indagine su di essa.

Christine JÉRUSALEM illustra la figura di Bergounioux che emerge dai suoi Carnets de notes. Lo stile di vita dello scrittore vi appare contraddistinto da una grande sobrietà, da un'estrema concentrazione e dall'onnipresenza dei libri, cui egli sembra chiedere di illuminare il senso della propria esistenza, accompagnata da una profonda malinconia.

Sylviane coyault analizza a sua volta i Carnets de notes, nel contesto dell'intera opera narrativa di Bergounioux. Catherine/Cathy ne emerge così come la sola figura femminile presente nella vita e nell'opera dello scrittore, una donna forte e combattiva che affronta il presente con vigore, determinazione e gioia di vivere, al punto di ergersi quale sintesi perfetta tra il mondo millenario degli antenati e quello della modernità, il cui caotico disordine è finalmente dominato, in virtù della cultura.

Il contributo di Éric LANGLOIS e Laurent RIEUTORT mira a ridisegnare i confini geografici e sociologici entro i quali si colloca l'opera di Bergounioux. In essa, l'antico sistema agropastorale, basato sui latifondi e sulla mezzadria, è colto nella fase della profonda crisi, causata dell'esodo massiccio verso le città che, a partire dal primo Novecento, spinge i contadini della Corrèze e del Limosino a ricorrere - come alcuni personaggi di Bergounioux - a tutta la creatività di cui sono capaci per non lasciare la loro terra.

21 Nel suo saggio, Liana NISSIM analizza la dimensione spaziale che tanta parte occupa nei romanzi dello scrittore limosino. Se il referente costante di Pierre Bergounioux è costituito dalla regione compresa tra Brive, Vézère e la Haute Corrèze, il paesaggio descritto dall'autore assume sovente sembianze umane o animali, suscitando, nello sguardo attento e meticoloso del narratore, il malinconico stupore per un mondo ormai irrimediabilmente perduto, che rende tuttavia onnipresenti le ombre degli antenati.

Marinella TERMITE si interessa alla tavolozza cromatica dispiegata da Bergounioux. Più varia nei romanzi, essa si limita a soli quattro colori nel breve testo Couleurs. Tuttavia in esso, il nero, il bianco e il grigio - dominanti nelle illustrazioni di Joël Leick che lo accompagnano - hanno il compito di richiamare alla memoria frammenti del passato, in un gioco di continui rimandi tra opera narrativa e opera critica. 
23 In seno alla produzione di Bergounioux, Guy LARROUx rintraccia quale cronotopo costante l'oscurità che invade al tempo stesso il paesaggio e l'interiorità del soggetto, fino ad abbracciare la storia dei suoi avi. Mentre l'aurora occupa, nei romanzi dell'autore, il punto più remoto e anteriore, la luce vi appare quasi miracolosamente, al termine di un lungo e faticoso processo di riflessione sull'esperienza, finalmente compresa dal narratore grazie alla mediazione dello studio e della scrittura.

Il volume si conclude con tre interventi che vedono, a diverso titolo, protagonista Pierre Bergounioux. Nella sua intervista, Matteo MAJORANO interroga il romanziere circa il valore della scrittura che per lui continua a costituire una delle più alte forme della domanda di senso di cui è capace l'uomo moderno.

Michel C. THомAS ripercorre poi la parabola creativa di Bergounioux, mostrandone la vastissima cultura, che abbraccia ambiti, autori, epoche e continenti diversi, coniugati però in una sintesi originale nelle opere dello scrittore.

Infine, Paul DE SALVANDY si sofferma sulle tre rivoluzioni che, secondo Bergounioux, hanno cambiato il corso della storia umana. La prima è la scoperta della scrittura, la seconda è l'invenzione della stampa e la terza riguarda la tecnologia applicata alla comunicazione, ed è quella che stiamo vivendo. 